\title{
Propofol attenuates intestinal mucosa injury induced by intestinal ischemia-reperfusion in the rat
}

\author{
[Le propofol atténue les lésions de la muqueuse intestinale provoquées par \\ l'ischémie-reperfusion intestinale chez le rat]
}

Ke-Xuan Liu PhD MD, ${ }^{*} \dagger$ Timo Rinne PhD MD, $\ddagger$ Wei He PhD MD, $\$$ Fang Wang MD MSc, $†$ Zhengyuan Xia PhD MD†

Purpose: We investigated whether propofol at a sedative dose can prevent intestinal mucosa ischemia/reperfusion (I/R) injury, and if propofol can attenuate oxidative stress and increases in nitric oxide (NO) and endothelin-I (ET-I) release that may occur during intestinal I/R injury.

Methods: Rats were randomly allocated into one of five groups ( $n=10$ each): (i) sham control; (ii) injury (one hour superior mesenteric artery occlusion followed by three hours reperfusion); (iii) propofol pre-treatment, with propofol given $30 \mathrm{~min}$ before inducing intestinal ischemia; (iv) simultaneous propofol treatment, with propofol given 30 min before intestinal reperfusion was started; (v) propofol post-treatment, with propofol given $30 \mathrm{~min}$ after intestinal reperfusion was initiated. In the treatment groups, propofol $50 \mathrm{mg} \cdot \mathrm{kg}^{-1}$ was administrated intraperitoneally. Animals in the control and untreated injury groups received equal volumes of intralipid (the vehicle solution of propofol) intraperitoneally. Intestinal mucosa histology was analyzed by Chiu's scoring assessment. Levels of lactic acid (LD), NO, ET-I, lipid peroxidation product malondialdehyde (MDA) and superoxide dismutase (SOD) activity in intestinal mucosa were determined.

Results: Histological results showed severe damage in the intestinal mucosa of the injury group accompanied by increases in MDA, NO and ET-I and a decrease in SOD activity. Propofol treatments, especially pre-treatment, significantly reduced Chiu's scores and levels of MDA, NO, ET-I and LD, while restoring SOD activity.

Conclusion: These findings indicate that propofol attenuates intestinal $\mathrm{I} / \mathrm{R}$-induced mucosal injury in an animal model. The response may be attributable to propofol's antioxidant properties, and the effects of inhibiting over-production of $\mathrm{NO}$ and in decreasing ET-I levels.

CAN J ANESTH $2007 / 54: 5 /$ pp 366-374

Objectif: Nous avons cherché à savoir si le propofol, en dose sédative, pouvait empêcher les lésions d'ischémie/reperfusion $(I / R)$ de la muqueuse intestinale, et s'il pouvait atténuer le stress oxydatif et les augmentations dans la libération d'oxyde nitrique (NO) et d'endothéline-I (ET-I) pouvant survenir lors de lésions I/R intestinales.

Méthode : Des rats ont été randomisés en cinq groupes $(n=10$ chacun) : (i) faux témoin (sham control); (ii) lésion (occlusion de l'artère mésentérique supérieure d'une heure suivie par reperfusion de trois heures) ; (iii) prétraitement au propofol, avec administration de propofol 30 min avant de provoquer l'ischémie intestinale; (iv) traitement simultané au propofol, avec administration de propofol 30 min avant le début de la reperfusion intestinale ; (v) traitement ultérieur au propofol, avec administration de propofol $30 \mathrm{~min}$ après le début de la reperfusion intestinale. Dans les groupes de traitement, du propofol a été administré en dose intrapéritonéale de $50 \mathrm{mg} \cdot \mathrm{kg}^{-1}$. Les animaux des groupes témoin et lésions nontraitées ont reçu des volumes équivalents d'Intralipid (la solution véhicule du propofol) en dose intrapéritonéale. L'histologie de la muqueuse intestinale a été analysée par l'évaluation des points de Chiu (Chiu's scoring assessment). Les niveaux d'acide lactique

From the Department of Anesthesiology, ${ }^{*}$ The First Affiliated Hospital, Sun Yat-sen University, Guangzhou, China; the Anesthesiology Research Laboratory, Department of Anesthesiology, $\uparrow$ Renmin Hospital, Wuhan University, Wuhan, Hubei, China; the Division of Cardiac Anesthesia and Intensive Care, $\ddagger$ Heart Center, Tampere University Hospital, Tampere, Finland; and the Department of Anesthesiology, $\mathbb{\$}$ Guangdong Provincial People's Hospital, Guangzhou, China.

Address correspondence to: Dr. Ke-Xuan Liu, Dept. of Anesthesiology, The First Affiliated Hospital, Sun Yat-sen University, Guangzhou, China. E-mail: liukexuan807@yahoo.com.cn. Or to: Dr. Zhengyuan Xia, Anesthesiology Research Laboratory, Dept. of Anesthesiology, Renmin Hospital of Wuhan University, 238 Jiefang Road, Wuhan, 430060, China. Phone: +86 27 88041919; Fax: +86 27 88042292; E-mail: zhengyuan_xia@yahoo.com. Current address: University of Calgary, Dept of Pharmacology \& Therapeutics, Calgary, Alberta, Canada.

Supported, in part, by a grant from Natural Science Foundation of Guangdong, China (No: 05300758, to K.X. Liu) and, in part, by grants from National Natural Science Foundation of China (No: 3067202 l, to K.X. Liu, and No: 30471659, to Z. Xia).

Accepted for publication January 15, 2007.

Revision accepted February 8, 2007. 
(LD), NO, ET-I, l'activité de produits de péroxydation lipidique de malondialdéhyde (MDA) et de superoxyde dismutase (SOD) dans la muqueuse intestinale ont été déterminés.

Résultats: Les résultats histologiques ont montré des lésions graves de la muqueuse intestinale dans le groupe lésions, accompagnées d'une augmentation de MDA, NO et ET-I et une diminution de l'activité SOD. Les traitements au propofol, particulièrement le prétraitement, ont réduit de façon significative les résultats et niveaux de MDA, NO, ET-I et LD sur l'échelle de Chiu, tout en restaurant l'activité SOD.

Conclusion: Ces résultats indiquent que le propofol atténue les lésions de la muqueuse intestinale provoquée par I/R chez le modèle animal. La réaction peut être attribuée aux propriétés anti-oxydantes du propofol, ainsi qu'aux effets d'inhibition de la surproduction de NO et de la diminution des niveaux de ET-I.

I NTESTINAL ischemia/reperfusion (I/R) injury is a serious condition which may result from hemorrhagic, traumatic or septic shock, or severe burns, and certain surgical procedures including small bowel transplantation, abdominal aortic surgery and cardiopulmonary bypass $(\mathrm{CPB}){ }^{1}$ It is well known that intestinal $\mathrm{I} / \mathrm{R}$ not only leads to the injury of intestine itself, but may also cause multiple organ dysfunction owing to damage of the intestinal mucosal barrier. ${ }^{2}$ Of particular interest, compromised peripheral perfusion during $\mathrm{CPB}$ and the resulting gastrointestinal mucosal injury have been shown to lead to decreased mucosal barrier function, which may allow translocation of intestinal flora and endotoxemia and subsequently increased systemic inflammation. ${ }^{3,4}$ This may lead to and/or further enhance oxidative stress during $\mathrm{CPB}$ and result in more eventful postoperative myocardial functional recovery.

The mechanisms of intestinal mucosa injury after intestinal $\mathrm{I} / \mathrm{R}$ are complex. Reactive oxygen species (ROS)-induced lipid peroxidation is known to be one of the major factors causing intestinal $\mathrm{I} / \mathrm{R}$ injury, and the administration of free radical scavengers appears to prevent intestinal mucosa from intestinal I/R injury. ${ }^{5}$ We have recently shown that antioxidant intervention during cardiac surgery under $\mathrm{CPB}$ attenuated gastric and intestinal mucosa injury and resulted in ameliorated postoperative myocardial injury, ${ }^{6}$ suggesting that antioxidant intervention can attenuate intestinal $I / R$ injury in the clinical setting. Propofol is an $i v$ anesthetic with antioxidant properties ${ }^{7,8}$ that is commonly used during cardiac surgery and postoperative sedation. ${ }^{9-11}$ Propofol has been shown to enhance tissue antioxidant capacity in various tissues in a rat model. ${ }^{12}$
Interestingly, low-dose propofol sedation attenuates the formation of ROS in tourniquet-induced ischemia-reperfusion injury in humans. ${ }^{13}$ It is unknown, however, whether propofol at a sedative dose can attenuate intestinal I/R-induced increase in oxidant stress and intestinal mucosal injury.

It has been reported that over-production of nitric oxide (NO) in intestinal mucosa tissue following intestinal $\mathrm{I} / \mathrm{R}$ can aggravate lipid oxidative damage $^{14,15}$ and that an increase of endothelin- 1 (ET-1) is involved in the pathogenesis of intestinal I/R-induced intestinal mucosal injury. ${ }^{16,17}$ Therefore, the current study was undertaken to clarify whether propofol can prevent intestinal mucosa I/R injury, and to investigate its effects on NO, ET-1 release during intestinal $\mathrm{I} / \mathrm{R}$, in an in vivo rat model. The accumulation of lactic acid (LD), a product from glucose metabolism in anaerobic metabolism, was used as an indirect index of intestinal ischemia.

\section{Methods}

Animal model

The current study was approved by the Animal Care Committee of Sun Yat-sen University, China and was performed in accordance with National Institutes of Health guidelines for the use of experimental animals. Fifty adult pathogen-free male Wistar rats weighing between 230 and $302 \mathrm{~g}$, were housed in individual cages in a temperature-controlled room with alternating $12 \mathrm{hr}$ light/dark cycles, and acclimated for one week before the study. Food was removed eight hours prior to the study, but all animals had free access to water.

All animals were anesthetized with pentobarbital (30 $\mathrm{mg} \cdot \mathrm{kg}^{-1}$ body weight, intraperitoneally), and the small intestine was exteriorized by midline laparotomy. The intestinal $\mathrm{I} / \mathrm{R}$ injury was established by occluding the superior mesenteric artery (SMA) with a microvessel clip for $60 \mathrm{~min}$ followed by $180 \mathrm{~min}$ reperfusion, as reported by Mitsuoka et al. ${ }^{18}$ Ischemia was recognized by the existence of pulseless or pale colour of the small intestine. The return of pulses and the re-establishment of the pink colour were assumed to indicate valid reperfusion of the intestine.

\section{Experimental protocol}

The rats were randomly allocated into one of the five groups ( $n=10$ per group): (i) control group (Control), in which sham surgical preparation including isolation of the SMA without occlusion was performed; (ii) injury group (Injury), in which intestinal I/R was produced by clamping SMA for one hour followed by declamping (i.e., reperfusion) for three hours; (iii) 
Propofol pre-treatment group (Pre-Prop), in which propofol was given $30 \mathrm{~min}$ before intestinal ischemia was induced; (iv) simultaneous propofol treatment group (Simu-Prop), in which propofol was given 30 min before intestinal reperfusion was started; (v) posttreatment group (Post-Prop), in which propofol was given $30 \mathrm{~min}$ after intestinal reperfusion was started. In the treatment groups, propofol (Diprivan, propofol 1\%, CG411, AstraZeneca, Caponago, Italy) 50 $\mathrm{mg} \cdot \mathrm{kg}^{-1}$ was administrated intraperitoneally. Animals in the control and injury groups received an equal volume of intralipid (vehicle solution of propofol) by $i p$ injection. The dose of propofol (i.e., $50 \mathrm{mg} \cdot \mathrm{kg}^{-1}$ ip) was chosen based on a preliminary experiment. This experiment showed that propofol $50 \mathrm{mg} \cdot \mathrm{kg}^{-1}$ $i p)$, a dose which inhibits rat hippocampal acetylcholine release to a lesser extent than does propofol 100 $\mathrm{mg} \cdot \mathrm{kg}^{-1} i{ }^{1},{ }^{19}$ produced a sedative response in rats, as determined by loss of reflex responses to a painful stimulus (needle skin prick), while remaining sensitive to skin incision. As propofol $60 \mathrm{mg} \cdot \mathrm{kg}^{-1}$ ip, provides satisfactory anesthesia in rats, ${ }^{20}$ we selected a slightly lower dose based on our preliminary study. Also, during the preliminary experiment, we found that neither intralipid nor physiological saline influenced the extent of intestinal mucosal damage in the injury group. Therefore, only intralipid was used as a vehicle control in the ensuing studies.

\section{Preparation of specimens}

After the completion of the experiments, the rats were killed with an iv overdose of pentobarbital sodium. A segment of $0.5-1.0 \mathrm{~cm}$ intestine was cut from $5 \mathrm{~cm}$ to terminal ileum, fixed in $4 \%$ formaldehyde polymerisatum, and embedded in paraffin for preparation. Another segment of small intestine was washed with cold saline and the intestinal mucosa was gently scraped off, dried with suction paper, and preserved at $-70^{\circ} \mathrm{C}$.

\section{Histological measurement of intestinal mucosal injury} The segment of small intestine was stained with hematoxylin-eosin. Damage of intestinal mucosa was initially evaluated independently by two pathologists who were blinded to the study groups. The degree of injury was evaluated using a modified Chiu's method ${ }^{21}$ according to changes of the villus and glands of the intestinal mucosa. The Chiu's score was graded as: 0 , normal villus and gland; 1 , changes at the top of villus and initial formation of subepidermal Gruenhagen's antrum; 2, formation of subepidermal Gruenhagen's antrum and slightly injured gland; 3 , enlargement of subepidermal gap and engorgement of capillary vessel;
4, epidermis moderately isolated with lamina propria and injured gland; 5 , top villus shedding; 6 , obvious villus shedding and capillary vessel dilating; 7 , lamina propria villus shedding, and distinct injured gland; 8 , initially decomposed lamina propria; 9, hemorrhage and ulceration. A minimum of six randomly chosen fields from each rat were evaluated and averaged to determine mucosal damage.

\section{Detection of lipid peroxidation and superoxide dis- mutase activity in intestinal mucosa}

Intestinal mucosal tissues were homogenized on ice with normal saline, frozen in a refrigerator at $-20^{\circ} \mathrm{C}$ for five minutes and centrifuged for $15 \mathrm{~min}$ at 4000 g. Supernatants were transferred into fresh tubes for the evaluation. The lipid peroxidation product malonedialdehyde (MDA) was measured by chemical analysis (Assay kits was supplied by Nanjing Jiancheng Biological Product, Nanjing, China) as previously described. ${ }^{22,23}$ The results were calculated as nmol.100 $\mathrm{mg}^{-1}$ tissue. Superoxide dismutase (SOD) activity was evaluated by inhibition of nitroblue tetrazolium reduction by superoxide anion generated by the xanthine/xanthine oxidase system using a commercial assay kit (Nanjing Jiancheng Biological Product, Nanjing, China) as described. ${ }^{22,23}$ The results were expressed as U.100 $\mathrm{mg}^{-1}$ protein.

\section{Detection of NO level in intestinal mucosa}

Intestinal mucosal tissues $(100 \mathrm{mg})$ were weighed and made into $10 \%$ homogenate with $0.9 \mathrm{~mL}$ physiological saline. After centrifugation for ten minutes at $10000 \mathrm{~g}$, the supernatant was placed in boiling water for three minutes and then centrifuged for five minutes at $10000 \mathrm{~g}$. Supernatant $(0.1 \mathrm{~mL})$ was taken for analysis using a commercial assay kit (Nanjing Jiancheng Biological Product, Nanjing, China). Nitrate and nitrite (NOx) were measured as oxidized stable end products of $\mathrm{NO}$ and the total nitrite level in the sample was determined according to the method described by Miranda et al. ${ }^{24}$ Results were calculated as $\mu \mathrm{mol} \cdot 100 \mathrm{mg}^{-1}$ protein.

\section{Detection of ET-1 level in intestinal mucosa}

Endothelin-1 level was measured by enzyme linked immunoassay (ELISA) techniques (assay kit was supplied by Beijing East Asian Radioimmunoassay Technology Institute, Beijing, China) as previously described. ${ }^{23}$ Briefly, $100 \mathrm{mg}$ intestinal mucosal tissue was boiled in $1 \mathrm{~mL}$ of a mixture of $1 \mathrm{M}$ acetate and 20 $\mathrm{mM}$ hydrochloride for ten minutes at $100^{\circ} \mathrm{C}$, and then centrifuged at $10000 \mathrm{~g}$ for ten minutes at $4^{\circ} \mathrm{C}$. The supernatant was filtrated, lyophilized, and dissolved 

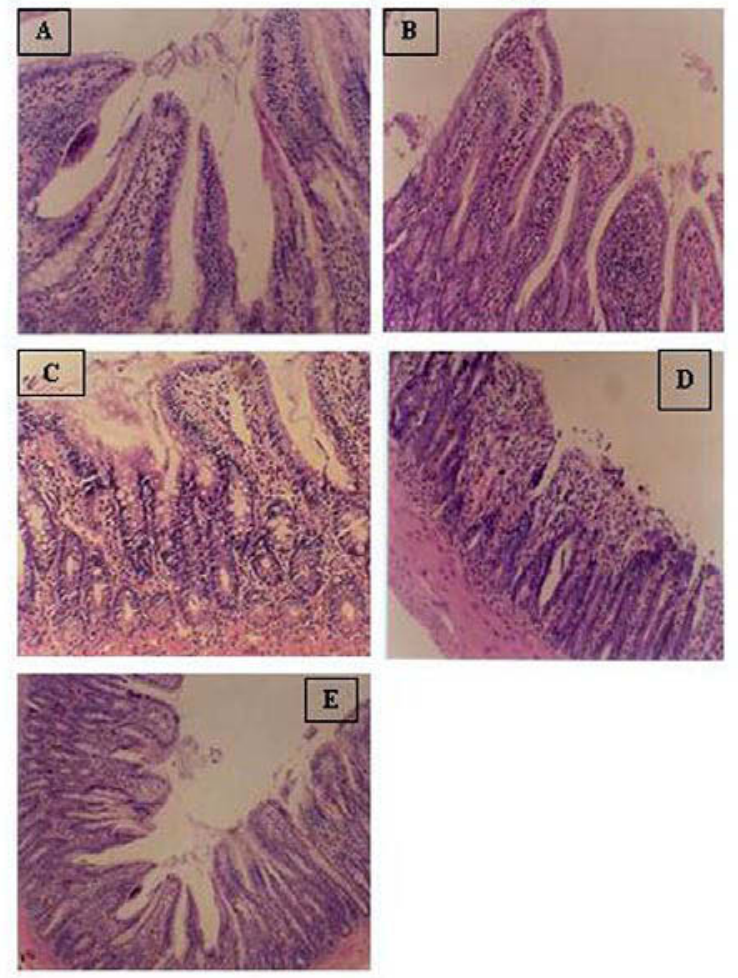

FIGURE 1 Histopathological changes of small intestine under light microscopy (× 200) A) In the control group, normal intestinal mucosa was seen. B) In the injury group, intestinal mucosa was damaged severely as shown by marked edema of mucosal villi and infiltration of necrotic epithelial and inflammatory cells. A large number of intestinal villi were severed and the gap between epithelial cells increased significantly. C) In the pre-treatment group, the damage was slight without significant edema. D) In the simultaneous treatment group, slight edema could be seen in intestinal villi, and some intestinal villi were severed. The gap between epithelial cells increased slightly. E) In the posttreatment group, a small number of intestinal villi were severed, and the increased gap between epithelial cells could be seen.

in $300 \mathrm{~mL}$ of buffer solution. This extracted peptide solution was applied to the ELISA plate. Endothelin1 level in samples was determined using a standard curve generated from known concentrations of ET-1. All measurements were performed in triplicate, and the intra- and interassay variability were $<10 \%$. Results were calculated as $\mathrm{pg} 100 \mathrm{mg}^{-1}$ protein.

\section{Detection of LD level in intestinal mucosa}

Intestinal mucosal tissues were weighed and made into $10 \%$ homogenate. The LD content in tissues was deter- mined using a chemical assay kit (Nanjing Jiancheng Biological Product, Nanjing, China) as described. ${ }^{25}$ The results were expressed as $\mathrm{mmol} \cdot \mathrm{g}^{-1}$ protein.

\section{Statistical analysis}

Statistics were analyzed with SPSS 11.0 software (SPSS Inc., Chicago, IL, USA). Data are expressed as mean \pm SD. One-way analysis of variance was used for multiple comparisons and the least significant difference test was used for intra-group comparison. Correlation between different variables was assessed by Spearman's coefficient, and $P<0.05$ was considered statistically significant.

\section{Results \\ Histological changes of intestinal mucosa under light microscopy}

As shown in Figure 1, in the control group, the villi and glands were normal and no inflammatory cell infiltration was observed in the mucosal epithelial layer (Figure 1A). In the injury group, severe edema of mucosal villi and infiltration of necrotic epithelial and inflammatory cells were observed, and intestinal glands showed evidence of mild injury. In addition, a large number of intestinal villi were severed, the gap of epithelial cells increased significantly, and blood and lymph vessels expanded markedly, indicative of severe mucosal damage (Figure lB). In the pre-treatment group, no significant edema and necrotic mucosal villi were seen, indicating that the damage was very minimal (Figure 1C). In the simultaneous treatment group, slight edema could be seen in intestinal villi, and some intestinal villi were severed. Intestinal glands could be seen in some specimens, and the gap between epithelial cells increased slightly (Figure ID). In the post-treatment group, a large number of intestinal villi were severed, and an increased gap between epithelial cells could be seen in severely damage areas, and blood and lymph vessels were expanded slightly (Figure 1E).

\section{Evaluation of intestinal mucosal injury}

As shown in the Table, Chiu's scores in the injury groups were significant higher than scores in the control group $(P<0.01)$. Compared with the injury group, Chiu's scores in the three treatment groups were significantly decreased (all $P<0.01$ ), but all scores exceeded those observed in the control group $(P<0.01)$. Chiu's score in the pre-treatment group was markedly lower $(P<0.01)$ than values observed in the simultaneous and post-treatment groups, suggesting that pre-treatment with propofol is better than other treatment regimens. 
TABLE Effects of propofol on SOD activity and the MDA, LD, NOx and ET-1 levels in intestinal mucosa

\begin{tabular}{|c|c|c|c|c|c|c|}
\hline Groups & Chiu's score & $\begin{array}{l}M D A \\
\left(\text { nmol.100 } \mathrm{m}^{-1}\right)\end{array}$ & $\begin{array}{l}\text { SOD } \\
\left(U \cdot 100 \mathrm{~m}^{-1}\right)\end{array}$ & $\begin{array}{l}\text { NOx } \\
\left(\mu m o l \cdot 100 \mathrm{mg}^{-1}\right)\end{array}$ & $\begin{array}{l}\text { ET-1 } \\
\left(\mathrm{mg} P g \cdot 100 \mathrm{mg}^{-1}\right)\end{array}$ & $\begin{array}{l}L D \\
\left(m m o \cdot g^{-1}\right)\end{array}$ \\
\hline Control & $0.98 \pm 0.74$ & $37.80 \pm 5.10$ & $40.82 \pm 5.07$ & $41.27 \pm 8.60$ & $284.12 \pm 46.1$ & $2.71 \pm 0.65$ \\
\hline Injury & $9.15 \pm 3.62$ * & $82.76 \pm 20.34^{*}$ & $24.75 \pm 9.70^{*}$ & $84.36 \pm 12.53^{*}$ & $691.50 \pm 109.98^{*}$ & $4.21 \pm 0.93^{*}$ \\
\hline Pre-Prop & $3.67 \pm 1.82 * \dagger$ & $35.72 \pm 9.24 \dagger$ & $58.18 \pm 6.94^{*} \dagger$ & $51.24 \pm 9.84 \dagger$ & $278.43 \pm 26.15 \dagger$ & $2.99 \pm 0.31 \dagger$ \\
\hline Simu-Prop & 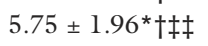 & $44.30 \pm 18.35 \dagger$ & $40.13 \pm 7.62 \dagger \ddagger$ & $60.41 \pm 15.89^{*} \dagger$ & $357.51 \pm 31.90^{* *} \dagger \ddagger \ddagger$ & $3.50 \pm 0.36 * * \dagger$ \\
\hline Post-Prop & $5.98 \pm 2.02^{*} \dagger \neq \ddagger$ & $62.13 \pm 16.67^{*} \dagger \ddagger ?$ & $39.32 \pm 8.43 \dagger \ddagger$ & $71.78 \pm 17.23^{*} \dagger \ddagger$ & $421.32 \pm 93.53 * \dagger \ddagger ?$ & $4.08 \pm 1.04^{* *} \ddagger$ \\
\hline
\end{tabular}

$\mathrm{SOD}=$ superoxide dismutase $\mathrm{MDA}=$ malondialdehyde $\mathrm{LD}=$ lactic acid $; \mathrm{NOx}=$ nitrate and nitrite; ET- 1 = endothelin- $\mathrm{l}$. Data are mean $\pm \mathrm{SD} ; n=10 .{ }^{*} P<0.01,{ }^{*} P<0.05$ vs the control group; $\dagger P<0.01$ vs the injury group; $\ddagger P<0.01, \ddagger \ddagger P<0.05$ vs the pre-treatment group (Pre-Prop); ?P $<0.05$ vs the simultaneous group (Simu-Prop).

\section{Changes of the MDA level and SOD activity in small intestinal mucosa}

As shown in the Table, the MDA level in the injury group was significantly higher than that in the control group $(P<0.01)$. Compared with the injury group, MDA levels in the three treatment groups were markedly reduced $(P<0.01)$. However, the MDA level in the post-treatment group was significantly higher than observed in the pre-treatment and the simultaneous groups $(P<0.01$ or 0.05 , Table $)$. In contrast, SOD activity in the injury group was significantly reduced $(P<0.05$, injury $v s$ control). Treatments with propofol markedly increased and restored SOD activity $(P$ $<0.01$, Pre-Prop, Simu-Prop or Post-Prop vs Injury, Table). Of interest, the SOD activity in the pre-treatment group was even higher than that observed in the control group $(P<0.05)$, and was also significantly higher than in other treatment groups $(P<0.01)$.

\section{Changes of the NOx and ET-I level in small intestinal mucosa}

As shown in the Table, the NOx level in the injury group was greater than in the control group $(P<$ $0.01)$. Nitric oxide levels in the three treatment groups were reduced as compared to the injury group $(P<0.01)$. The NO level in the pre-treatment group did not differ from that in the control group $(P>$ 0.05, Pre-Prop vs Control, Table) but was markedly lower than that observed in the post-treatment group $(P<0.01)$. Similarly, the level of ET-l in the injury group was increased as compared to the control group $(P<0.01)$. Compared with the injury group, the level of ET-1 was reduced by the treatments with propofol $(P<0.01)$. However, Pre-Prop, but not Simu-Prop or Post-Prop, restored ET-1 to the control value $(P>$ 0.05 , Pre-Prop vs Control). The ET-1 level in the PreProp group was lower than that in Post-Prop group $(P<0.01)$.

\section{Changes of the LD level in intestinal mucosa}

The LD level in the injury and post-treatment groups were significantly higher than in the control group $(P$ $<0.05$ ). Pre-Prop and Simu-Prop, but not Post-Prop, significantly reduced the increase of LD as compared to the injury group $(P<0.01)$ (Table).

\section{Correlation analysis}

Overall $(n=50)$, strong positive correlations between Chiu's score and MDA $(r=0.83, P<0.0001$, Figure $2 \mathrm{~A})$, between MDA and ET- $1(\mathrm{r}=0.89, P<0.0001$, Figure 2C) and between Chiu's score and NOx $(r=$ $0.87, P<0.0001$, Figure $2 \mathrm{D}$ ) were identified. In contrast, ET- 1 was inversely correlated to SOD activity ( $\mathrm{r}$ $=-0.78, P<0.0001$, Figure $2 \mathrm{~B})$. Also, strong positive correlations between MDA and NOx $(\mathrm{r}=0.83, P<$ 0.0001 , Figure 2E) as well as between NOx and ET-1 $(\mathrm{r}=0.77, P<0.0001$, Figure $2 \mathrm{~F})$ were identified.

\section{Discussion}

We have demonstrated in a rat model that one hour occlusion of the SMA followed by three hours of reperfusion caused significant intestinal $\mathrm{I} / \mathrm{R}$ injury as evidenced by pathological morphological changes and increased Chiu's scores seen in the intestinal mucosa, which is in accordance with a previous report. ${ }^{5}$ The intestinal $\mathrm{I} / \mathrm{R}$ injury was associated with dramatic increases in the intestinal mucosa, of MDA, ET-1, $\mathrm{NO}$ and LD, and a decrease in SOD activity. The novel finding of the current study is that propofol, at a sedative dosage, significantly attenuated SMA occlusion - reperfusion induced intestinal mucosal damage and the above mentioned biochemical changes when given either prior to, during SMA occlusion, or during the early phase of reperfusion. Most intriguingly, propofol pretreatment normalized changes of MDA, ET-1, NO and LD and stimulated an over-production 

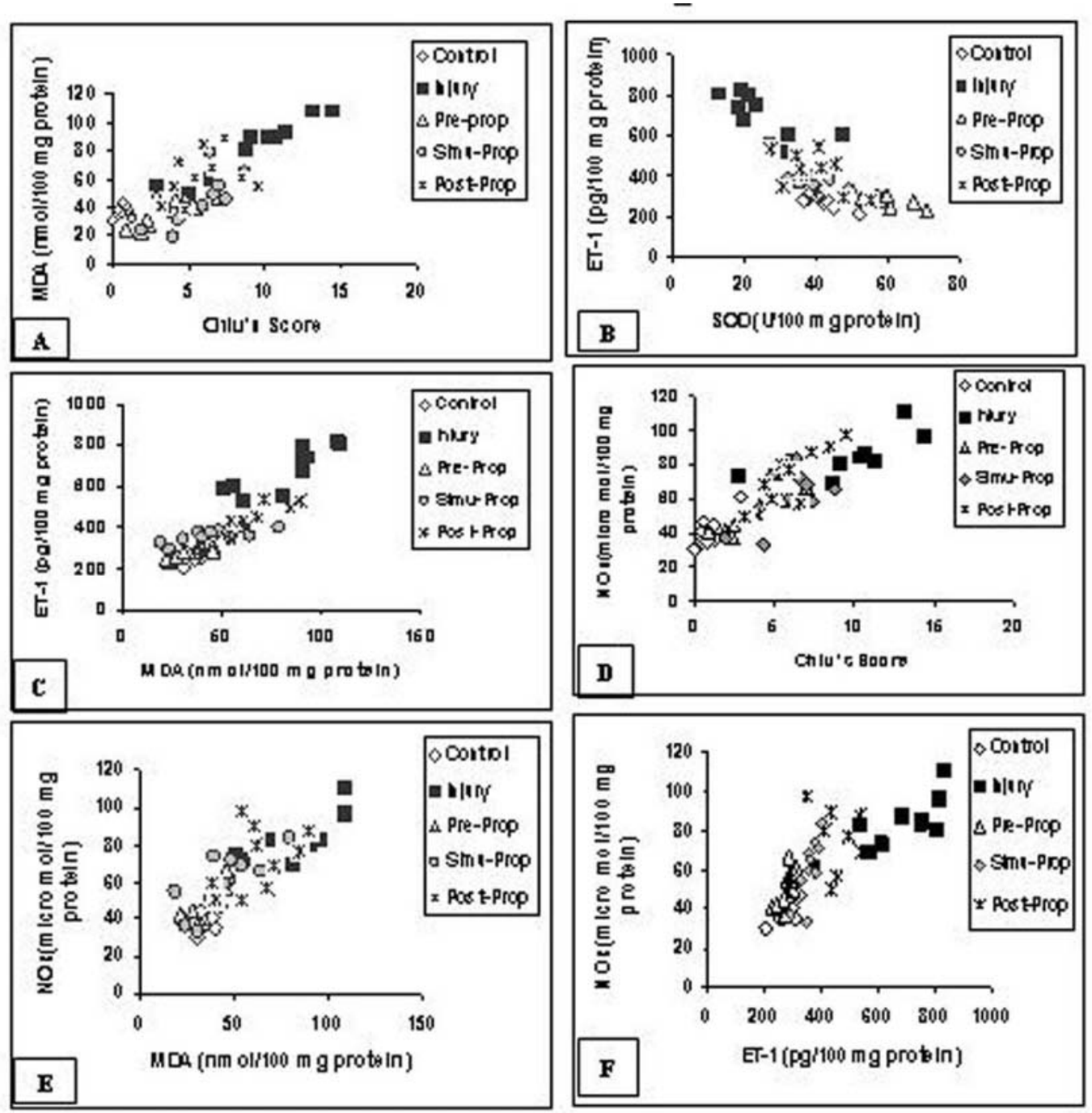

FIGURE 2 Correlations between Chiu's score and malonedialdehyde (MDA) (A), between endothelin (ET)-1 and superoxide dismutase (SOD) (B) or MDA (C), between Chiu's score and NOx production (D), between NOx production and MDA (E), and between NOx production and ET-1 (F). Strong positive correlations between Chiu's score and MDA ( $\mathrm{r}=$ $0.83,95 \%$ confidence interval: $0.71-0.90, P<0.0001, A)$, between MDA and ET- $1(\mathrm{r}=0.89,95 \%$ confidence interval: 0.81-0.94, $P<0.0001, C)$, between Chiu's score and NOx $(r=0.87,95 \%$ confidence interval: $0.78-0.92, P<0.0001, \mathrm{D})$, between NOx and MDA $(\mathrm{r}=0.83,95 \%$ confidence interval: $0.71-0.90, P<0.0001, \mathrm{E})$, and between NOx and ET-1 $(\mathrm{r}=$ $0.77,95 \%$ confidence interval: $0.63-0.87, P<0.0001, \mathrm{~F})$, were identified. In contrast, ET-1 was inversely correlated with SOD activity $(\mathrm{r}=-0.78,95 \%$ confidence interval: -0.87 to $-0.64, P<0.0001, \mathrm{~B})$.

of endogenous SOD (Table). Intracellular SOD has been shown to play a critical role in attenuating the intestinal inflammatory response. ${ }^{26,17}$

Propofol is widely used as an anesthetic before and during cardiac surgery, and as a sedative postoperatively in the intensive care unit. Also, in the clinical setting, pre-treatment with a certain drug for diseases related to intestinal $\mathrm{I} / \mathrm{R}$ injury occurs with a higher frequency compared to other regimens, but sometimes treatment may be initiated after the onset of ischemia or during reperfusion due to unexpected occurrence of $\mathrm{I} / \mathrm{R}$ event. In this regard, three different propofol regimens were used in the present study and a sedative dose of propofol was chosen to 
investigate its effect on intestinal mucosal injury after intestinal I/R. Our results show that every propofol treatment regimen could significantly alleviate postischemic intestinal mucosal injury. However, propofol pre-treatment conferred the most profound protective effect. This is suggestive of a preconditioning-like effect of propofol, at least in the intestine. Ischemic preconditioning refers to a phenomenon in which a tissue is rendered resistant to the deleterious effects of prolonged ischemia by previous exposure to brief periods of vascular occlusion, and this preconditioning effect can be mimicked by pharmacological agents. Indeed, propofol has been shown to significantly increase heme oxygenase production in astrocytes and astroglial cells. ${ }^{27,28}$ Heme oxygenase is a molecule with antioxidant properties that has been demonstrated to play a critical role in intestinal ischemic preconditioning that mediates protection against intestinal mucosal injury and the subsequent systemic inflammatory response..$^{29,30}$ Therefore, propofol may have initiated a preconditioning-like effect that is characterized by an increase of the endogenous antioxidant defenses, such as the increase of heme oxygenase and SOD activities. This is intriguing and merits further study.

It is known that oxidant stress is one of major factors contributing to intestinal $\mathrm{I} / \mathrm{R}$ injury. ${ }^{5,31}$ In our study, intestinal $\mathrm{I} / \mathrm{R}$ injury was associated with a significant decrease of SOD activity, a major endogenous antioxidant enzyme, and increase of the lipid peroxidation product MDA in the injury group. Treatment with propofol increased SOD activity and attenuated MDA production that was associated with a reduced Chiu's score (Table). The significant positive correlation between tissue MDA content and Chiu's score (Figure 2A) is consistent with the notion that lipid peroxidation is a major cause of post-ischemic intestinal injury. A positive correlation between MDA and ET-1 (Figure 2C) as well as between MDA and NO suggests that the increase in lipid peroxidation is attributable, in part, to the increases of NO and ET1. A recent study by Yagmurdur et al. ${ }^{32}$ shows that propofol, but not the iv anesthetic ketamine, prevents burn injury induced increase in lipid peroxidation and attenuates gut mucosal epithelial apoptosis in rats, an effect that may be attributable to propofol antioxidant properties.

Although NO produced through constitutive NO synthase can be an important protective molecule for the small intestine at the onset of intestinal $\mathrm{I} / \mathrm{R},{ }^{15}$ over-production of $\mathrm{NO}$ through the inducible $\mathrm{NO}$ synthase (iNOS), especially under the circumstance of oxidant stress, may prove detrimental. The tight positive correlation between Chiu's score and NO production (Figure 2D) is consistent with the notion that over-production of $\mathrm{NO}$ could be detrimental. Under oxidant stress, the concurrent formation of high levels of superoxide and NO favour their reaction to form the potent oxidant peroxynitrite, resulting in further increased oxidative as well as nitrosative stress. Inhibition of iNOS has been shown to prevent the increase of NO production, reduce lipid peroxidation and attenuate intestinal $\mathrm{I} / \mathrm{R}$ injury in the rats. ${ }^{14,33}$ Propofol has been shown to suppress NO biosynthesis by inhibiting iNOS expression in lipopolysaccharideactivated macrophages ${ }^{34}$ and inhibit the over-production of $\mathrm{NO}$, leading to reduced vascular superoxide production and attenuated endothelial dysfunction in septic rats. ${ }^{35}$ Further, propofol can react with peroxynitrite to form a propofol-derived phenoxyl radical, and therefore function as a peroxynitrite scavenger. ${ }^{8}$ Although the effects of propofol on iNOS expression and phenoxyl radicals were not investigated in the present study, propofol inhibition of NO production (Table) suggests that its protective effect against intestinal I/R injury may be associated with the suppression of iNOS-NO-peroxynitrite pathway.

Endothelin-1 is an important participant in ischemia-reperfusion induced cardiovascular complications. Increased ET-l activity is not only a causative factor to intestinal $\mathrm{I} / \mathrm{R}$ injury, ${ }^{17,36}$ but most importantly, elevated plasma ET-1 levels might be related to the size and extent of myocardial infarction and the mortality after myocardial infarction in patients, ${ }^{37}$ a situation that is often accompanied with gastrointestinal complications. ${ }^{38,39}$ Propofol attenuation of ET-1 production in the injured intestinal mucosa (Table) could potentially reduce its release to the circulation. It may also represent a mechanism whereby propofol attenuates MDA formation (Table), since ET-1 has been shown to stimulate superoxide production. ${ }^{40}$ In addition, propofol reduction of the mucosa LD level, an index of anaerobic glucose metabolism, is indicative of improved intestinal mucosal microcirculation, which may be attributable to its effect in reducing ET- 1 , a potent vasoconstrictor.

In conclusion, we have shown that treatment, especially pre-treatment, with propofol at a sedative dose attenuates intestinal $\mathrm{I} / \mathrm{R}$-induced intestinal mucosa injury in an animal model. Further work is required to determine if this response translates to the bedside when propofol is used for conscious sedation following major cardiac surgery or for critical care patients at risk for gastrointestinal ischemia. The current results lend support to our previous hypothesis that propofol sedation might add to the beneficial effect of volatile anesthetic preconditioning. ${ }^{41}$ Finally, in interpreting 
these data, we caution that the intestinal ischemiareperfusion insult that was examined in the current study is more severe than that which might be caused by $\mathrm{CPB}$. In addition, although propofol is mainly absorbed into blood circulation after ip injection, it remains to be determined whether ip injection of propofol $50 \mathrm{mg} \cdot \mathrm{kg}^{-1}$ could have produced a substantially larger concentration in the intestinal mucosa than would have resulted from a smaller dose of propofol administrated intravenously. Further studies in different animal models and in particular, in the clinical setting, are warranted.

\section{Acknowledgements}

The authors are very grateful to Dr. You-Kai Zhu and Dr. Chui-Liang Liu for their help in the histological measurement and replication of the animal model.

\section{References}

1 Mallick IH, Yang W, Winslet MC, Seifalian AM. Ischemia-reperfusion injury of the intestine and protective strategies against injury. Dig Dis Sci 2004; 49: 1359-77.

2 Farhadi A, Banan A, Fields J, Keshavarzian A. Intestinal barrier: an interface between health and disease. J Gastroenterol Hepatol 2003; 18: 479-97.

3 Mollhoff T, Loick HM, Van Aken H, et al. Milrinone modulates endotoxemia, systemic inflammation, and subsequent acute phase response after cardiopulmonary bypass (CPB). Anesthesiology 1999; 90: 72-80.

4 Riddington DW, Venkatesh B, Boivin CM, et al. Intestinal permeability, gastric intramucosal $\mathrm{pH}$, and systemic endotoxemia in patients undergoing cardiopulmonary bypass. JAMA 1996; 275: 1007-12.

5 Riaz AA, Wan MX, Schafer T, et al. Allopurinol and superoxide dismutase protect against leucocyte-endothelium interactions in a novel model of colonic ischaemia-reperfusion. Br J Surg 2002; 89: 1572-80.

6 Xia ZY, Liu XY, Zhan LY, He YH, Luo T, Xia Z. Ginsenosides compound (shen-fu) attenuates gastrointestinal injury and inhibits inflammatory response after cardiopulmonary bypass in patients with congenital heart disease. J Thorac Cardiovasc Surg 2005; 130 : 258-64.

7 Murphy PG, Myers DS, Davies MJ, Webster NR, Jones $J G$. The antioxidant potential of propofol (2,6-diisopropylphenol). Br J Anaesth 1992; 68: 613-8.

8 Mathy-Hartert M, Mouithys-Mickalad A, Kohnen S, Deby-Dupont G, Lamy $M$, Hans P. Effects of propofol on endothelial cells subjected to a peroxynitrite donor (SIN-1). Anaesthesia 2000; 55: 1066-71.

9 Xia Z, Huang Z, Ansley DM. Large-dose propofol during cardiopulmonary bypass decreases biochemi- cal markers of myocardial injury in coronary surgery patients: a comparison with isoflurane. Anesth Analg 2006; 103: 527-32.

10 De Hert SG, Crombeecke S, ten Broecke PW, et al. Effects of propofol, desflurane, and sevoflurane on recovery of myocardial function after coronary surgery in elderly high-risk patients. Anesthesiology 2003; 99: 314-23.

11 Bovill JG. Intravenous anesthesia for the patient with left ventricular dysfunction. Semin Cardiothorac Vasc Anesth 2006; 10: 43-8.

12 Runzer TD, Ansley DM, Godin DV, Chambers GK. Tissue antioxidant capacity during anesthesia: propofol enhances in vivo red cell and tissue antioxidant capacity in a rat model. Anesth Analg 2002; 94: 89-93.

13 Cheng YJ, Wang YP, Chien CT, Chen CF. Small-dose propofol sedation attenuates the formation of reactive oxygen species in tourniquet-induced ischemia-reperfusion injury under spinal anesthesia. Anesth Analg 2002; 94: 1617-20.

14 Naito $\Upsilon$, Takagi T, Ichikawa H, et al. A novel potent inhibitor of inducible nitric oxide inhibitor, ONO1714 , reduces intestinal ischemia-reperfusion injury in rats. Nitric Oxide 2004; 10: 170-7.

15 Cuzzocrea S, Chatterjee PK, Mazzon E, et al. Role of induced nitric oxide in the initiation of the inflammatory response after postischemic injury. Shock 2002; 18: 169-76.

16 Massberg $S$, Boros M, Leiderer R, Baranyi L, Okada $H$, Messmer K. Endothelin (ET)-1 induced mucosal damage in the rat small intestine: role of $\mathrm{ET}(\mathrm{A})$ receptors. Shock 1998; 9: 177-83.

17 Oktar BK, Gulpinar MA, Bozkurt A, et al. Endothelin receptor blockers reduce $\mathrm{I} / \mathrm{R}$-induced intestinal mucosal injury: role of blood flow. Am J Physiol Gastrointest Liver Physiol 2002; 282: G647-55.

18 Mitsuoka H, Unno N, Sakurai T, et al. Pathophysiological role of endothelins in pulmonary microcirculatory disorders due to intestinal ischemia and reperfusion. J Surg Res 1999; 87: 143-51.

19 Inagawa G, Sato K, Kikuchi T, et al. Chronic ethanol consumption does not affect action of propofol on rat hippocampal acetylcholine release in vivo. $\mathrm{Br} \mathrm{J}$ Anaesth 2004; 93: 737-9.

20 Brasil LJ, San-Miguel B, Kretzmann NA, et al. Halothane induces oxidative stress and NF-kappaB activation in rat liver: protective effect of propofol. Toxicology 2006; 227: 53-61.

21 Chin CJ, McArdle AH, Brown R, Scott HJ, Gurd FN. Intestinal mucosal lesion in low-flow states. I. A morphological, hemodynamic, and metabolic reappraisal. Arch Surg 1970; 101: 478-83.

22 Luo T, Xia Z. A small dose of hydrogen peroxide 
enhances tumor necrosis factor-alpha toxicity in inducing human vascular endothelial cell apoptosis: reversal with propofol. Anesth Analg 2006; 103: 110-6.

23 Xia Z, Gu J, Ansley DM, Xia F, Yu J. Antioxidant therapy with Salvia miltiorrhiza decreases plasma endothelin- 1 and thromboxane B2 after cardiopulmonary bypass in patients with congenital heart disease. J Thorac Cardiovasc Surg 2003; 126: 1404-10.

24 Miranda KM, Espey $M G$, Wink DA. A rapid, simple spectrophotometric method for simultaneous detection of nitrate and nitrite. Nitric Oxide 2001; 5: 62-71.

25 Chang L, Du JB, Gao LR, Pang YZ, Tang CS. Effect of ghrelin on septic shock in rats. Acta Pharmacol Sin 2003; 24: 45-9.

26 Kruidenier L, van Meeteren ME, Kuiper I, et al. Attenuated mild colonic inflammation and improved survival from severe DSS-colitis of transgenic $\mathrm{Cu} / \mathrm{Zn}$ SOD mice. Free Radic Biol Med 2003; 34: 753-65.

27 Acquaviva R, Campisi A, Murabito P, et al. Propofol attenuates peroxynitrite-mediated DNA damage and apoptosis in cultured astrocytes: an alternative protective mechanism. Anesthesiology 2004; 101: 1363-71.

28 Acquaviva R, Campisi A, Raciti G, et al. Propofol inhibits caspase- 3 in astroglial cells: role of heme oxygenase-1. Curr Neurovasc Res 2005; 2: 141-8.

29 Ferencz A, Szanto Z, Borsiczky B, et al. The effects of preconditioning on the oxidative stress in small-bowel autotransplantation. Surgery 2002; 132: 877-84.

30 Tamion F, Richard V, Lacoume $\Upsilon$, Thuillez C. Intestinal preconditioning prevents systemic inflammatory response in hemorrhagic shock. Role of HO-1. Am J Physiol Gastrointest Liver Physiol 2002; 283: G40814.

31 Stefanutti G, Pierro A, Vinardi S, Spitz L, Eaton S. Moderate hypothermia protects against systemic oxidative stress in a rat model of intestinal ischemia and reperfusion injury. Shock 2005; 24: 159-64.

32 Yagmurdur $H$, Aksoy $M$, Arslan M, Baltaci B. The effects of propofol and ketamine on gut mucosal epithelial apoptosis in rats after burn injury. Eur J Anaesthesiol 2007; 24: 46-52.

33 Kubes P, McCafferty DM. Nitric oxide and intestinal inflammation. Am J Med 2000; 109: 150-8.

34 Chen RM, Wu GJ, Tai YT, et al. Propofol reduces nitric oxide biosynthesis in lipopolysaccharide-activated macrophages by downregulating the expression of inducible nitric oxide synthase. Arch Toxicol 2003; 77: 418-23.

35 Yu HP, Lui PW, Hwang TL, Yen CH, Lau YT.

Propofol improves endothelial dysfunction and attenuates vascular superoxide production in septic rats. Crit Care Med 2006; 34: 453-60.

36 Nankervis CA, Schaner GM, Miller CE. Endothelin- mediated vasoconstriction in postischemic newborn intestine. Am J Physiol Gastrointest Liver Physiol 2000; 279: G683-91.

37 rasuda $M$, Kobno $M$, Tabara A, et al. Circulating immunoreactive endothelin in ischemic heart disease. Am Heart J 1990; 119: 801-6.

38 Mangi AA, Christison-Lagay ER, Torchiana DF, Warshaw AL, Berger DL. Gastrointestinal complications in patients undergoing heart operation: an analysis of 8709 consecutive cardiac surgical patients. Ann Surg 2005; 241: 895-901; discussion 901-4.

39 Omland T, Lie RT, Aakvaag A, Aarsland T, Dickstein $K$. Plasma endothelin determination as a prognostic indicator of 1-year mortality after acute myocardial infarction. Circulation 1994; 89: 1573-9.

40 Pollock DM, Pollock JS. Endothelin and oxidative stress in the vascular system. Curr Vasc Pharmacol 2005; 3 : 365-7.

41 Xia Z, Luo T. Sevoflurane or desflurane anesthesia plus postoperative propofol sedation attenuates myocardial injury after coronary surgery in elderly high-risk patients. Anesthesiology 2004; 100: 1038-9. 